\title{
L'œuvre de Raymonde April: une écriture de la félicité
}

\author{
Christine Palmiéri
}

L'œuvre de Raymonde April et la misogynie; paradoxe ou provocation? C'est poser sur l'un des plateaux d'une balance douceur et sérénité et, sur l'autre, haine et violence. Peut-on associer des actants si opposés pour questionner la relation complexe entre masculinité et féminité?

La misogynie, tout comme le racisme, issu de frustrations, de rancœurs personnelles ou de préjugés collectifs, a pour caractéristiques, dans le besoin d'épancher la douleur, la colère, la haine, d'identifier une seule et même cible, l'autre, dans l'irrationalité d'une généralisation aveugle. À travers les siècles, de multiples images se superposent devant les yeux des misogynes, qu'ils plaquent inconsciemment sur le visage de femmes anonymes. Sans en faire l'historique, rappelons au passage l'image de son infériorité originaire dans la religion chrétienne - excroissance d'une côte masculine - puis celle du péché originel - la fameuse pomme. Elle sera qualifiée de souillure, puis d'hystérique possédant des forces maléfiques, de sorcière manipulatrice, de frivole, de libertine, de mère trop protectrice ou autoritaire et distante, puis de femme de pouvoir depuis le XXe siècle.

On le voit bien, tout tourne autour de la relation passionnelle, dans sa dimension de domination ou de servitude. Il en résulta des mesures irrationnelles et cruelles, comme la chasse aux sorcières, la lapidation, l'enfermement, l'excision ou le port du voile obligatoire dans certaines cultures et religions.

Cette obéissance à laquelle la femme est astreinte, pour satisfaire la domination-possession mâle, est régie par des règles et des lois dans certaines communautés, alors que les Occidentaux ont accepté en apparence l'égalité entre les deux sexes favorisant l'émancipation féminine. Certaines dérives tragiques en résultent. L'ultime: les tueurs en série. Terreur et horreur sont les images extrêmes de la misogynie que donnent à voir ces déviants, ces êtres gouvernés par la haine qui pointent le canon de leur fusil sur des femmes anonymes, étranglent, dépècent, violent, cachent les restes des corps comme de la barbaque envenimée sinon vénéneuse. Les femmes sembleraient empoisonner l'existence de certains qui ne peuvent réprimer leur pulsion vengeresse. Tous les misogynes heureusement n'ont pas la même intensité de colère et se contentent souvent de miner la vie des femmes dans leur entourage immédiat ou lointain, allant jusqu'à nuire à leur sécurité ou à leur carrière professionnelle.

À l'opposé de ces figures de femmes maléfiques, pécheresses, souillées, dominatrices ou légères, l'œuvre de Raymonde April porte un regard tout en douceur sur le monde des hommes et des femmes, un " regard amoureux » 
qui caresse les contours du quotidien dans un travail ou la technique s'estompe devant le naturel des situations pour permettre une adhésion totale au rythme ondulant des paysages et des personnages dans des cercles familiaux, amicaux, ou dans une solitude sereine.

Les photographies donnent à voir, une femme, des femmes, des hommes aussi, des paysages extérieurs et intérieurs. Un journal intime, comme de nombreux artistes entretiennent, mais, à l'inverse d'eux, elle le fait sans provocation comme chez Sophie Calle, qui exhibe ses multiples relations amoureuses, ou chez Rodney Graham, qui tourne la vie en dérision, ou encore chez Michel Gerson, qui héroïse l'anti-héros. L'auteur nous offre son monde avec l'innocence d'un œil capteur d'une réalité toute en lumière. Un univers sans ombre. Dans une totale nudité. Sans retenue ou bien si habilement camouflée. Des portraits dans la simplicité des postures spontanées. Rien de grandiloquent. Un monde tel quel, un monde de grâce. Raymonde April révèle le côté éclairé des choses, la lumière qui enrobe les rondeurs du monde. Un monde personnel sans artifice et sans fiction qui nous enserre dans ses bras, nous freine devant l'intrépidité de la vie, nous berce dans une rêverie mnésique.

Mais comment fonctionnent symboliquement ces photos qui nous transportent dans un état de parfaite harmonie avec le monde, un état de félicité? Prenons la photo intitulée Quatre amis. Dans un cadre champêtre, quatre hommes se laissent voir et «capturés» par l'objectif de l'artiste dans un moment de repos. Scène du quotidien rarement utilisée comme sujet d'une œuvre, manifestant de la tendresse envers un enfant et de l'amitié entre eux. D'eux d'entre eux discutent, très attentifs l'un à l'autre, le troisième paresse tendrement. Leur tenue vestimentaire et les taches de peinture sur les jeans et les mains de celui qui occupe le premier plan nous renseignent sur la nature de leur repos. Ils ne sont pas en vacances, ils travaillent ensemble. Rien d'exceptionnel, sinon le fait de les avoir cadrés, d'en avoir fait le sujet d'une œuvre, d'avoir montré des hommes dans des attitudes loin des stéréotypes de macho ou de séducteur, dans un jeu de rôle appris. Au contraire, ils ont l'air naturels, calmes et sincères, dans des attitudes singulières.

Dans Deux horizons, l'intérieur s'ouvre vers l'extérieur, vers l'horizon que la femme guette, toujours tournée qu'elle est vers l'avenir, inquiète et curieuse, alors que l'homme profite de la sécurité du cocon familial. Sécurité et insécurité semblent être les deux pôles qui régissent cette image si habituelle des comportements de couple.

Dans Échinacée, rien de plus étonnant de voir un homme parmi les fleurs dans un sous-bois, rien dans l'histoire ne nous prépare à cette scène masculine bucolique. Même si le personnage cherchait à placer des pièges à lièvre, ou à commettre une action illicite, le rendu duveteux des buissons, des arbres feuillus et la couleur des fleurs créent une atmosphère attendrissante.

Dans Miroir, la fenêtre s'ouvre cette fois sur l'appartement de l'artiste où une femme, sa mère, le regard affectueux, offre son profil aux rayons de soleil, d'une ville, Paris? Que l'artiste semble vouloir pointer de son objectif, vu le nombre d'équipements photographiques jonchant la table au premier plan.

On peut se demander, devant ces images, si l'artiste occulte le côté sombre du monde pour ne pas le voir, ne pas être troublée, indifférente aux malheurs 
du monde, ou si elle cherche à révéler comment voir les petits bonheurs qui nous échappent, pour s'imprégner de sagesse, pour mieux affronter le mal et dialoguer avec la misère. La colère ne résout rien, et l'indignation ne peut trouver de solution que dans le calme et la réflexion sensée, semblent dire ces images.

Ces cuvres ont un pouvoir exutoire, en effet. Elles apaisent et nous réconcilient avec le monde, nous font apprécier cette banalité des jours qui est riche de paix. Message de paix, voilà ce qu'elles nous offrent, nous apprennant à regarder, à estimer ces moments fugitifs qui sont comme des baumes sur nos douleurs, à jouir de ce qui nous entoure, nous qui sommes des privilégiés. Nous qui pouvons agir pour aider et protéger les plus démunis et les victimes. Reconstruire nos valeurs. Cette ouverture, cette nudité et cette simplicité dérangent, car cela ne correspond pas aux images stéréotypées de femmes diaboliques et dominatrices. L'artiste ne cherche pas à impressionner ou à choquer par une imagination débridée, ne fait pas étalage d'une poïétique démesurée. Elle propose une sorte d'esthétique de l'interstice.

Ces hommes, ces femmes, corps dans l'espace du familier, dessinent l'univers du quotidien, l'univers de mémoire où filiation, parenté et amitié en tissent les mailles virtuelles. Si l'auteure ne figure plus sur les photos, comme ce fut le cas dans des productions antérieures, elle y est plus que présente par l'intérêt qu'elle porte à ses proches, à ses intérieurs-extérieurs. Elle en fait des icônes, de la matière-lumière, de la matière d'ombre. Cuvres sensibles avec lesquelles le spectateur «empathise» dans une relation intersubjective inconsciente. Comme s'il s'en émanait une fluidité secrète, magnétique et organique à la fois. C'est sur le principe de percept immanent ou encore de « percept sublime » (voir Michel Onfray) que fonctionnent ces ouvres et pas seulement sur le mode d'affects mnésiques.

Il est évident que devant le monde en furie qui lutte, se bat à coup de bombes, parfois humaines explosives et implosives, dans des pays de feu et de glace, en surface ou en souterrain que rien ne semble calmer, l'artiste, loin de nier les tragédies du monde, oppose à l'horreur des conflits et des actes criminels la sérénité de certaines relations humaines en symbiose avec leur environnement, sans excès, sans passion fougueuse. Elle creuse le réel pour mieux habiter le présent comme dans une sorte de rituel, dans une quête de purification des affects. Elle ne donne pas à voir l'horreur et le nihilisme du siècle et tout ce qui tourmente les femmes, la violence sexiste, les pathologies sociales ou encore les pratiques culturelles discriminatoires en Chine et dans les pays musulmans, les féminicides du Mexique ou les gynécides d'Occident, formes ultimes de la misogynie actuelle. Mais elle en montre souvent l'isolement, l'esseulement, notamment dans la série Inconscientes, titre qui brouille les pistes de la signification, ouvre la voix à une nouvelle logique.

D'ailleurs dans Inconscientes. Aigle et Inconscientes. Evans, l'artiste semble mettre en scène ces occultations repérées auparavant en une dramatisation insolite manifestée par les voiles et les jeux de lumière. Par les silhouettes esseulées, les intérieurs et extérieurs réunis en des espaces cette fois fragmentés puis réunifiés. Dans Inconscientes. Loup, une menace semble troubler les univers 
de paix auxquels elle nous a habitués. Un homme s'éloigne, des loups avancent rageusement, les architectures basculent dans un chaos graphique. Soudain la lumière baisse, la clarté s'estompe, le trouble, le flou jaillissent. Même si l'écriture reste épurée sur un mode minimal, sobre et dépouillée d'enflure, l'angoisse s'esquisse, s'immisce, le doute naît.

Cette ouvre participe-t-elle de la misogynie qui nous préoccupe ici? Peut-être par la liberté d'exprimer la douceur, la simplicité du quotidien, qui ne peut que frustrer les hommes habitués à réprimer leurs émotions liées au bonheur, et habitués à montrer leur violence et leur agressivité comme signe de leur virilité. Cet interdit que l'homme se voit imposer par l'éducation, avant tout masculine, à savoir de masquer sa féminité (tout ce qui renvoie aux gestes autorisés pour les femmes), peut faire haïr les femmes, celles-là mêmes qui ont le droit d'exprimer leurs états d'âme - mais c'est aussi tout ce qu'elles ont eu le droit de faire pendant longtemps.

Dans son travail de mise en relation des êtres humains et de la nature sans disharmonie, sans déséquilibre ni dissonance ni désordre, sans simulacre ni simulation, cette euvre opère un retour à l'immanence, geste apparemment infime mais immense en même temps, qui envahit notre corps et notre esprit d'un sentiment et d'une sensation de paix incroyable. A-t-on affaire à une nouvelle écriture? À une écriture du sacré? Une écriture de la félicité? Quel est ce pouvoir mystérieux qui émane de ces œuvres? Magie ou sorcellerie? On y revient, par d'autre voix. Au déclenchement de la haine! 